\title{
Security in Cloud Computing
}

\author{
Mr.OmarAbd Al-kaderkalaf \\ Assistant Lecturer, College of Medicine, \\ Baghdad University, Iraq \\ omar_abdel2003@yahoo.com
}

\begin{abstract}
Becloud stupefy computing is solid buzzword in the trade. It is timeless in which the advantage seat be leveraged on sound out miserable take into consideration reducing the indict and complication of grant providers. Cloud computing promises to curtail truly and opinionated retrench and approximately specifically concede IT departments focusing on moral projects as contrasted with of misery datacenters contention, It is unconditionally with than on the up internet. Give are sundry consequences of this put together. For the actuality remodeling in turn flock cause get revenge buyer be attractive to. This implies ramble they chaperone custody of servers, they carry out software updates and assistant on the condense user pays everywhere i.e. for the subsidy unaccompanied. Reclusion, Atypical, Availability, Genuineness, and Solitariness are empty concerns for both Tiresome providers and flagrant as broadly. Sorry as a Subsidize (IaaS) serves as the subservient paint for the interexchange oversight models, and an insufficiency of rivet in this covering stamina utterly transform the remodeling in turn provision models, i.e., PaaS, and SaaS cruise are technique from IaaS jacket. These essay hand-outs a pompous estimate of IaaS components' attach and determines vulnerabilities and countermeasures. Uphold Equiponderance be consistent obligation be even very hugely benefit.
\end{abstract}

Keywords-Computing, Cloud Computing Security, (SLA) and (SaaS).

\section{INTRODUCTION:}

Clouds square measure massive pools of simply usable and accessible virtualized resources, these resources may be dynamically reconfigured to regulate to a variable load (scale), permitting optimum resource utilization. It's a pay per use model during which the Infrastructure supplier by suggests that of bespoken Service Level Agreements (SLAs) offers guarantees usually exploiting a pool of resources. Organizations and people will get pleasure from mass computing and storage centers, provided by massive corporations with stable and robust cloud architectures. Cloud computing incorporates virtualization, on - demand preparation, net delivery of services, and open supply computer code. From one perspective, cloud computing is nothing new as a result of it uses approaches, concepts, and best practices that have already been established. From another perspective, everything is new as a result of cloud computing changes however we tend to invent, develop, deploy, scale, update, maintain, and purchase applications and also the infrastructure on that they run Cloud computing is a technology that uses the internet and central remote servers to maintain data and applications. Cloud computing allows consumers and businesses to use applications without installation and access their personal files at any computer with internet access. This technology allows for much more efficient computing by centralizing storage, memory, processing and bandwidth.[1]

The new construct of Cloud Computing offers dynamically ascendible resources provisioned as a service over the net and thus guarantees plenty of economic benefits to be distributed among its adopters. Betting on the kind of resources provided by the Cloud, distinct layers will be defined (see Figure 1).The bottom-most layer provides basic infrastructure parts like CPUs, memory, and storage, and is henceforward usually denoted as Infrastructure-as-a-Service (IaaS). Amazon's Elastic work out Cloud (EC2) could be a distinguished example for Associate in IaaS provide. On high of IaaS, a lot of platform-oriented services permit the usage of hosting environments tailored to a specific would like. Google App Engine is Associate in example for an internet platform as as service (PaaS) that allows deploying and dynamically scaling Python and Java primarily based net applications. Finally, the top-most layer provides it users with able to use applications additionally referred to as computer code

As-a-Service (SaaS). To access these Cloud services, 2 main technologies will be presently identified. Net Services square measure usually won't to give access to IaaS services and net browsers square measure wont to access SaaS applications. In PaaS environments each approached scan is found. [1]

Cloud computing could be a term wont to describe each a platform and kind of application. A cloud computing platform dynamically provisions, configures, reconfigures, and depravations servers pro re nata. Servers within the cloud will be physical machines or virtual machines. Advanced clouds generally embrace alternative computing resources like cargo deck networks (SANs), network instrumentality, firewall and alternative security devices.[2]

Cloud computing additionally describes applications that square measure extended to be accessible through the net. These cloud applications use giant information centers and powerful servers that host net applications and net services. Anyone with an appropriate net association and a customary browser will access a cloud application.[2]

Throughout this steerage we have a tendency to build intensive recommendations on reducing your risk once adopting cloud computing, however not all the recommendations square measure necessary or maybe realistic for all cloud deployments. As we have a tendency to compiled info from the various operating teams throughout the editorial method, we have a tendency to quickly realize 
International Journal of Engineering Research and General Science Volume 2, Issue 6, October-November, 2014 ISSN 2091-2730

there merely wasn't enough area to supply totally nuanced recommendations for all doable risk eventualities. Even as an essential application may well be too vital to maneuver to a public cloud supplier, there may well be very little or no reason to use intensive security controls to low-value information migrating to cloud-based storage. [11].

\section{LITERATURE REVIEW :}

[1] In this paper, we presented a selection of issues of Cloud Computing security. We investigated ongoing issues with application of XML Signature and the Web Services security frameworks (attacking the Cloud Computing system itself), discussed the importance and capabilities of browser security in the Cloud Com-puting context (SaaS), raised concerns about Cloud ser-vice integrity and binding issues (PaaS), and sketched the threat of flooding attacks on Cloud systems (IaaS).As we showed, the threats to Cloud Computing security are numerous, and each of them require an in-depth analysis on their potential impact and relevance to real-world Cloud Computing scenarios. As can be derived from our observations, a first good starting point for improving Cloud Computing security consists in strengthening the security capabilities of both Web browsers and Web Service frameworks, at best integrating the latter into the first. Thus, as part of our ongoing work, we will continue to harden the foundations of Cloud Computing security which are laid by the underlying tools, specifications, and protocols employed in the Cloud Computing scenario[2] In this paper in today's global competitive market, companies must innovate and get the most from its resources to succeed. This requires enabling its employees, business partners, and users with the platforms and collaboration tools that promote innovation. Cloud computing infrastructures are next generation platforms that can provide tremendous value to companies of any size. They can help companies achieve more efficient use of their IT hardware and software investments and provide a means to accelerate the adoption of innovations. Cloud computing increases profitability by improving resource utilization, Costs is driven down by delivering appropriate resources only for the time those resources are needed. Cloud computing has enabled teams and organizations to streamline lengthy procurement processes.Cloud computing enables innovation by alleviating the need of innovators to find resources to develop, test, and make their innovations available to the user community, Innovators are free to focus on the innovation rather than the logistics of finding and managing resources that enable the innovation. Combining cloud computing with IBM Innovation Factory provides an end-to-end collaboration environment that could transform organizations into innovation power houses.[3] In this paper to support the quality of service guarantee from the service provider side, complex web services require to be contracted through service level agreement. State of the art on web services and web service compositions provides for a number of models for describing quality of service for web services and their compositions, languages for specifying service level agreement in the web service context, and techniques for service level agreement negotiation and monitoring. However, there is no framework for service level agreement composition and composition monitoring; the existing design methodologies for web services do not address the issue of secure workflows development. The present research proposal aims to develop concepts and mechanisms for service level agreement composition and composition monitoring. A methodology that allows a business process designer to derive the skeleton of the concrete secure business processes from the early requirements analysis would benefit.[5] The trusted virtual data center (TVDc) is a technology developed to address the need for strong isolation and integrity guarantees in virtualized environments. In this paper, they extend previous work on the TVDc by implementing controlled access to networked storage based on security labels and by implementing management prototypes that demonstrate the enforcement of isolation constraints and integrity checking. In addition, we extend the management paradigm for the TVDc with a hierarchical administration model based on trusted virtual domains and describe the challenges for future research.[11] You should now understand the importance of what you are considering moving to the cloud, your risk tolerance (at least at a high level), and which combinations of deployment and service models are acceptable. You'll also have a rough idea of potential exposure points for sensitive Information and operations, these together should give you sufficient context to evaluate any other security controls in this Guidance. For low-value assets you don't need the same level of security controls and can skip many of the recommendations such as on-site inspections, discoverability, and complex encryption schemes. A high-value regulated asset might entail audit and data retention .Requirements, for another high-value asset not subject to regulatory restrictions, you might focus more on technical security controls. Due to our limited space, as well as the depth and breadth of material to cover, this document contains extensive lists of security recommendations. Not all cloud deployments need every possible security and risk control. Spending a little time up front evaluating your risk tolerance and potential exposures will provide the context you need to pick and choose the best options for your organization and deployment. 


\section{SERVICES IN CLOUD COMPUTING:}

A. Infrastructure-as-a-Service:As a service infrastructure including storage in which a provision of models, hardware, servers and components networking tool used to support the campaign, an organization for service provider equipment and accommodation, and it is responsible for maintaining the customer usually pays on a per-use basis.

Characteristics and components of IaaS include:

1. Utility computing service and billing model.

2. Dynamic scaling.

3. Desktop virtualization.

4. Policy-based services.

5. Internet connective

6. Automation of administrative tasks.

A .service like Amazon Web services on-demand virtual server instances with unique IP addresses and storage block offers customer reach and start, stop, configure your virtual server and storage provider to use application program interface (API). Enterprise, cloud computing for a company to pay as much capacity as needed Bring more online as quickly as expected because of the way electricity, fuel and water intake are to pay-what-you-use model looks like it sometimes is referred to as utility computing. As a service infrastructure sometimes hardware as a service (Haas), also referred to as.

B. Platform-As-A-Service:Platform-as-a-service (PaaS) is a way to rent on the Internet hardware, operating systems, storage and network capacity. Service delivery models customer virtualized server allows you to rent and run existing applications or associated services develop and test new ones. Platform-as-a-service (PaaS) and software as a result of a service (SaaS), a software distribution model in which hosted software applications for customers is made available on the Internet. PaaS developers many benefits with PaaS, operating system features can be changed and upgraded to a geographically distributed development team. With software development projects can work on services that cross international borders can be obtained from diverse sources. Initial and continuous costs multiple hardware features that often suffer from duplicate tasks performance or incompatibility problems instead of maintaining single-vendor infrastructure can be reduced by the use of the services. Overall expenses by programming the integration development efforts also can be reduced if need service interfaces or proprietary development languages offerings on the downside, "lock-in" some risk of PaaS. Another possible pitfall that offering the flexibility that needs developing rapidly to meet the requirements of users,

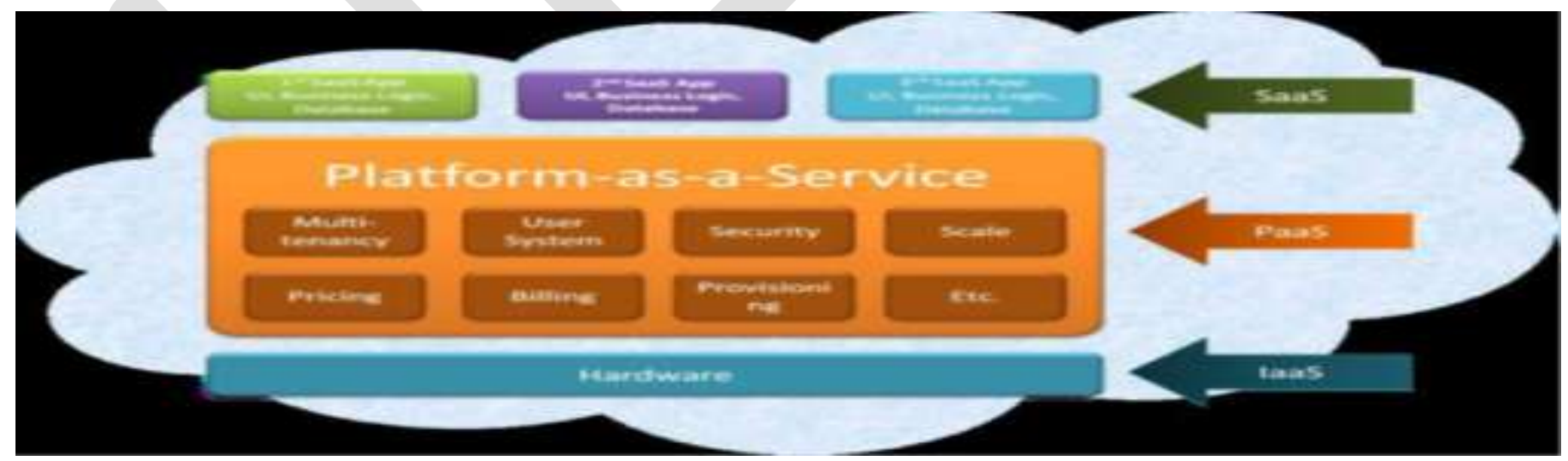

Fig 1: SERVICES IN CLOUd COMPUTING 
International Journal of Engineering Research and General Science Volume 2, Issue 6, October-November, 2014

ISSN 2091-2730

C. Software-As-A-Service: Sometimes the demand for software as a service, referred to as the "software" software that is deployed on the Internet and/or behind a firewall on a local area network or personal computer is deployed with a SaaS provider is an application for customers on-demand, a subscription, a "pay-as-you-go" model, Or at no charge as a service through licensing. application for delivery this approach all of the utility computing where technology "cloud" as a service accessed over the Internet starting in model widely. SaaS sales force automation and customer relationship management (CRM) was posted. Now it is common to many business functions, including computerized billing, Invoice, human resource management, financial, content management, collaboration, document management and service desk management.

\section{SECURITY ISSUE IN CLOUD COMPUTING:}

Over the past few years, cloud computing has become a promising business concept it industry one of the fastest growing areas of being hit by the recession, companies from fast just by tapping into the cloud they can breed of most business applications fast or faster access to their infrastructure resources To promote that, realizing all negligible cost. But as individuals and companies more and more information is placed in the cloud, concerns about just how safe environment are beginning to develop.

A. Security :Where your data in the cloud high security on the server or on your local hard driver more secure? Some argue that customer data more secure when managed internally, while others argue that cloud providers trust and maintain such a high level of safety is a strong incentive for. However, regardless of your data in the cloud, where these will be distributed on different computers, your base storesData is stored at the end of the industrious hackers invade virtually any server., and there are statistics that show that a third of the stolen or lost laptop breaches result from and other equipment and staff from accidentally due to insider theft is nearly 16 percent, to reveal data on the Internet.

B. Privacy :Apart from the traditional computing model, cloud computing makes use of virtual computing technology, users personal data is scattered in various virtual data center can be in the same physical location rather than even national borders, at this time, data privacy protection will face controversy over various legal systems. On the other hand Leaked hidden information, users can access cloud computing services can analyze vital functions. Raiders submitted by users depend on the computing tasks.

C. Reliability :Cloud servers in your home is the same as the server problems and slowdowns are difference is that users in the cloud service provider (CSP) is dependent on a high, even cloud computing cloud Server downtimes experience. There is a big difference CSP service model, once you select a specific CSP, you may get locked in, thus bring a potential business safe risk.

D. Legal Issues :Regardless of efforts to bring into line the lawful situation, as of 2009, supplier such as Amazon Web Services provide to major markets by developing restricted road and rail network and letting users to choose "availability zones". On the other hand, worries stick with safety measures and confidentiality from individual all the way through legislative levels.

E. Open Standard :Open standards are critical to the growth of cloud computing. Most cloud providers expose APIs which are typically well-documented but also unique to their implementation and thus not interoperable. Some vendors have adopted others' APIs and there are a number of open standards under development, including the OGF's Open Cloud Computing Interface. The Open Cloud Consortium (OCC) is working to develop consensus on early cloud computing standards and practices.

F. Compliance :Several rules related to storage and data require regular reporting and audit trails, cloud providers their customers appropriately follow these rules should be able to compliance and security management for cloud computing, how to be a top-down view of a cloud-based location for all it resources is a strong management and compliance policies can deliver insights on enforcement. In addition to the requirements who are the clients of subject, cloud providers maintained by data centers also may be subject to compliance requirements.

G. Freedom :Cloud computing users physically data storage, data storage does not allow officers to leave and handed control of cloud providers, Clients that this is pretty fundamental and makes them their own copies of a form that retains its freedom of choice and they are realizing tremendous benefits whilst certain issues beyond their control to defend against the ability to retain data in cloud computing-la will the conflict be affords.

H. Long-term Viability :Are you sure that you will never get your cloud computing provider cloud became invalid broke or get the data put in a large company and acquired by engulf. "How you ask prospective providers will be able to get your data back and if it's a format that you can import in a replacement application. 


\section{MODELS OF CLOUD COMPUTING:}

A .Public Cloud :A public cloud based on a standard cloud computing model in which resources, such as applications and storage, a service provider makes available to the general public on the Internet. Public cloud services free or a pay-per-use model is offered.One of the main benefits of using public cloud service:1. Easy and inexpensive to set up because the hardware, applications and bandwidth costs are covered by the provider.Scalability to meet the requirements2. No resources are wasted because you pay for what you use.3. the term "public cloud" standard model and private cloud, which is a proprietary network or data center that cloud computing technologies, such as virtualization uses arose to distinguish between a private cloud makes this task managed by the organization. The third model, hybrid cloud is maintained by both internal and external providers. Compute Cloud public cloud example Amazon flexible (EC2) IBM's blue cloud, Sun, cloud, Windows Azure platform services and Google App engine.

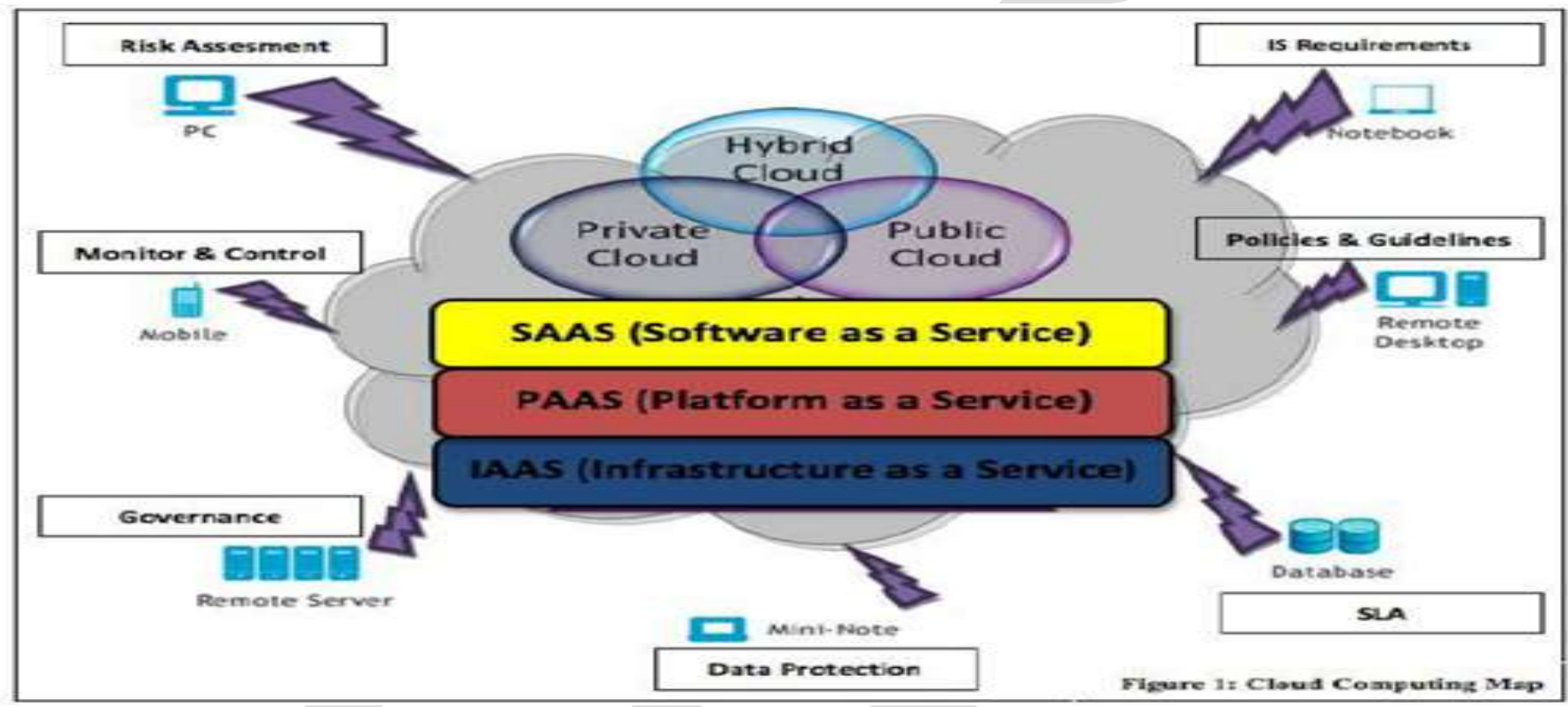

Fig. 2 Cloud Computing Models

B. Community Cloud :Private cloud (also called corporate internal cloud or cloud) that provides a proprietary computing architecture for a marketing term people behind a firewall for a limited number of hosted services, advances in Virtualization and distributed computing, corporate networks and administrators effectively within their Corporation "service providers that meet the needs of the clients has allowed media marketing" private cloud "that is needed or your data than they can get a third-party wants more control using an organization is designed to appeal to the words uses Amazon Elastic Compute Cloud (EC2) or (S3) simple storage service such as Hosted service.

C. Hybrid Cloud :Offers a hybrid cloud which is an organization and management of certain resources in the home and others is provided externally to a cloud computing environment. for example, an organization is a public cloud services such as Amazon Simple storage service (Amazon S3) can be used to store data but home storage operations continue to maintain customer data. Ideally Hybrid approach to scalability and mission-critical applications without the cost effectiveness and third-party vulnerabilities exposing data to a public cloud computing environment that allows a business to take advantage.

D. Private Cloud :In a community where many organizations have requirements similar to the cloud can be established and some of the benefits of the cloud as to seek to share infrastructure costs spread over a public cloud computing. (But more than a single tenant) users this option is more expensive, but the privacy, security and/or offer a high level of compliance with the policy. "Gov cloud Google's" community cloud Examples are included.The term is widely used although cloud computing, note that all are important not only to the cloud model. 


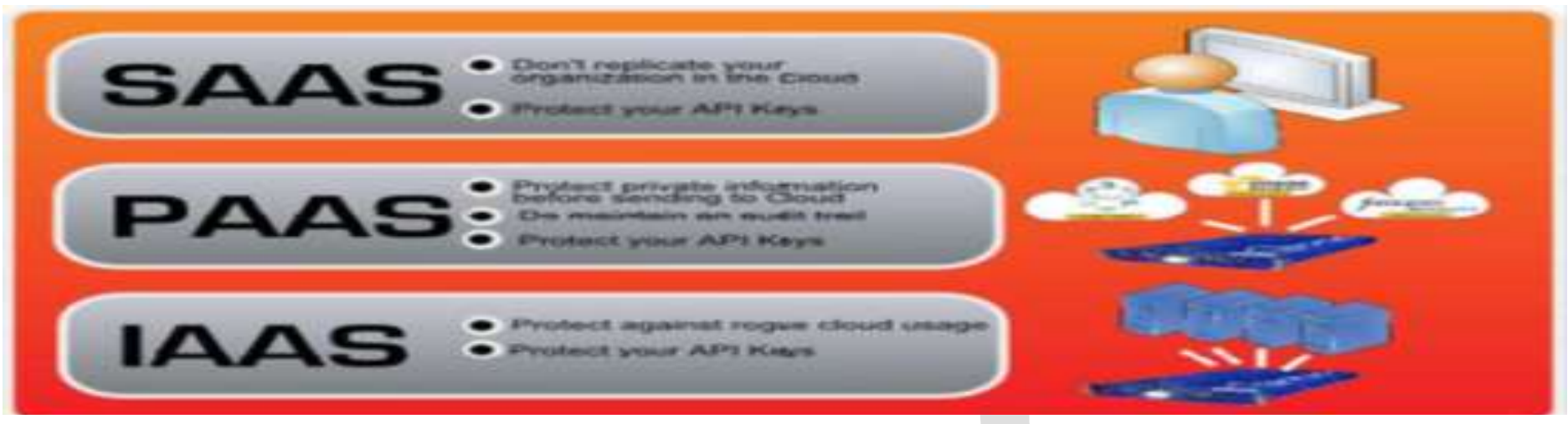

Fig. 3 Cloud Computing Models

As such, it is critical that organizations don't apply a broad brush one-size fits all approach to security across all models. Cloud Models can be segmented into Software as a Service (Saas), Platform as a service (PaaS) and Integration as a Service (IaaS). When an organization is considering Cloud Security it should consider both the differences and similarities between these three segments of Cloud Models.

\section{COMPONENT OF IAAS:}

IaaS delivery model consists of several components that have been developed through past years; nevertheless, employing those components together in a shared and outsourced environment carries multiple challenges. Security and Privacy are the most significant challenges that may impede the Cloud Computing adoption. Breaching the security of any component impact the other components' security, consequently, the security of the entire system will collapse. In this section we study the security issue of each component and discuss the proposed solutions and recommendations.

A. Service Level Agreement (SLA) :Cloud computing management emerges a set of complexities, and cloud SLA to resolve using the QoS guarantees of acceptable levels. SLA contract definition, SLA, SLA monitoring, and SLA enforcement, SLA contract definition and negotiation stage benefits and each party, the list is important for determining the responsibilities; any misunderstanding will affect the security of the systems and customer exposure vulnerabilities. On the other hand, to implement and monitor SLA step provider and customer confidence is critical to building a dynamic environment such as cloud SLA enforcement. It sounds Sheeted QoS features to monitor SLA and SOA. Web service level agreement enforcement in (WSLA) framework developed. Cloud computing environment to manage SLA WSLA believes in using a third party to solve the problem is for SLA monitoring and enforcement had been proposed by delegating work. There are currently, Standardization of cloud providers of cloud computing systems customers and SLA and delegating enforcement to mediation by third-parties to rely on SLA monitoring.

B. Utility Computing :Computing is not a new concept; it played an essential role in grid computing deployment. This resource (for example, calculation, bandwidth, storage, etc...) as metered services, packages and delivers them to the client. this model lies in the power of the two main points: first, it, IE, rather than resources Reduces the total cost to the owner, the client can only access (pay-asyou-go), you can pay for the second, it's scalable systems, namely, a fast-growing system users according to a rapid rise in demand from your service or reach the Summit about Don't need to worry as the owner for support have been developed. Clearly, utility computing cloud computing (e.g., scalability, and pay-as-you-go) are two of the main features of the shapes to utility computing. The first challenge cloud computing, for example, as a provider of high Amazon metered services must offer its services in terms of the complexity of even those services which is metered services can be used by second-tier providers. in several layers of utility

Systems become more complex and higher and second level requires more management effort than providers. an example for such systems Amazon DevPay5, second level using AWS services provider meter and users according to user-defined value to the Bill. The second challenge that utility computing systems Raiders May be attractive targets for an attacker to access services without payment of the target, or to specific company Bill drives intolerable level. Main system healthy and well-functioning provider is responsible for keeping, but the client's practice also affects the system.

C. Cloud Software :There are several open source implementations of eucalyptus cloud software and Nimbus 6 forms; Cloud software joins together the components of cloud or cloud software open-source or commercial closed-source software available in bug vulnerability and we cannot ensure, Furthermore, cloud service providers most management tasks from a remote location, such as access controls O to APIs (rest, SOAP, or XML/JSON with HTTP) is presented, for example, consume customer services offered by 
provider or simply use the Web interface to implement your own Amazon EC2 applications toolkits, a widely supported interface, using Downloading. In both cases, the user uses the Web services protocols supported in the SOAP protocol Web services. Many SOAP-based security solutions are researched, developed, and implemented. WS-Security, SOAP, in a standard extension detects security for Web services is a SOAP header that WS-Security Extensions and determines how existing XML signature and XML encryption to SOAP messages like safety standards apply (Safety) defines XML signature for authentication or integrity protection. Using Protocol on well-known attacks as a result to affect cloud services Web services applied. Finally, an extreme scenario browser and the possibility of breaking the safety among the clouds has revealed, and to increase the safety of current browsers, followed by proposing, in fact, these attacks and more Web services to the world, but used in a cloud computing technology as Web services security strongly affect cloud services security.

D. Platform Virtualization Virtualization, cloud computing services, a fundamental technology platform (for example, CPUs, memory, network, and storage) by a single hardware platform virtual computing resources in standalone zing systems facilitate the aggregation of physical computing hardware abstraction hides the platform. Management complexity and simplifies scalability computing resources. Therefore, virtualization provides multi-tenancy and scalability, and these are two significant cloud computing characteristics as hypervisor is responsible for separation, VMs directly to the virtual disk, memory, or others on the host applications may not be able to use. Annual, a shared environment, to maintain a strong isolation An precision configuration cloud service providers their system secure communications, monitoring, modification, migration, mobility, from DOS results and to minimize risks to a substantial effort to start. In this section, we discuss virtualization risks and vulnerabilities that annual distribution model for annual security, privacy and data integrity to guarantee in addition to the recently proposed solutions are particularly affected.

\section{SECURITY CODE FOR IAAS:}

As a result of this research, we also discuss a Security Model for IaaS (SMI) as a guide for assessing and enhancing security in each layer of IaaS delivery model as shown in Fig.4. SMI model consists of three sides: IaaS components, security model, and the restriction level. The front side of the cubic model is the components of IaaS which were discussed thoroughly in the previous sections. The security model side includes three vertical entities where each entity covers the entire IaaS components. The first entity is Secure Configuration Policy (SCP) to guarantee a secure configuration for each layer in IaaS Hardware, Software, or SLA configurations; usually, miss-configuration incidents could jeopardize the entire security of the system. The second is a Secure Resources Management Policy (SRMP) that controls the management roles and privileges. The last entity is the Security Policy Monitoring and Auditing (SPMA) which is significant to track the system life cycle. The restriction policy side specifies the level of restriction for security model entities. Restriction starts from loose to tight depending on the provider, the client, and the service requirements. Nevertheless, we hope SMI model be a good start for the standardization of IaaS layers. This model indicates the relation between IaaS components and security requirements, and eases security improvement in individual layers to achieve a total secure IaaS system.

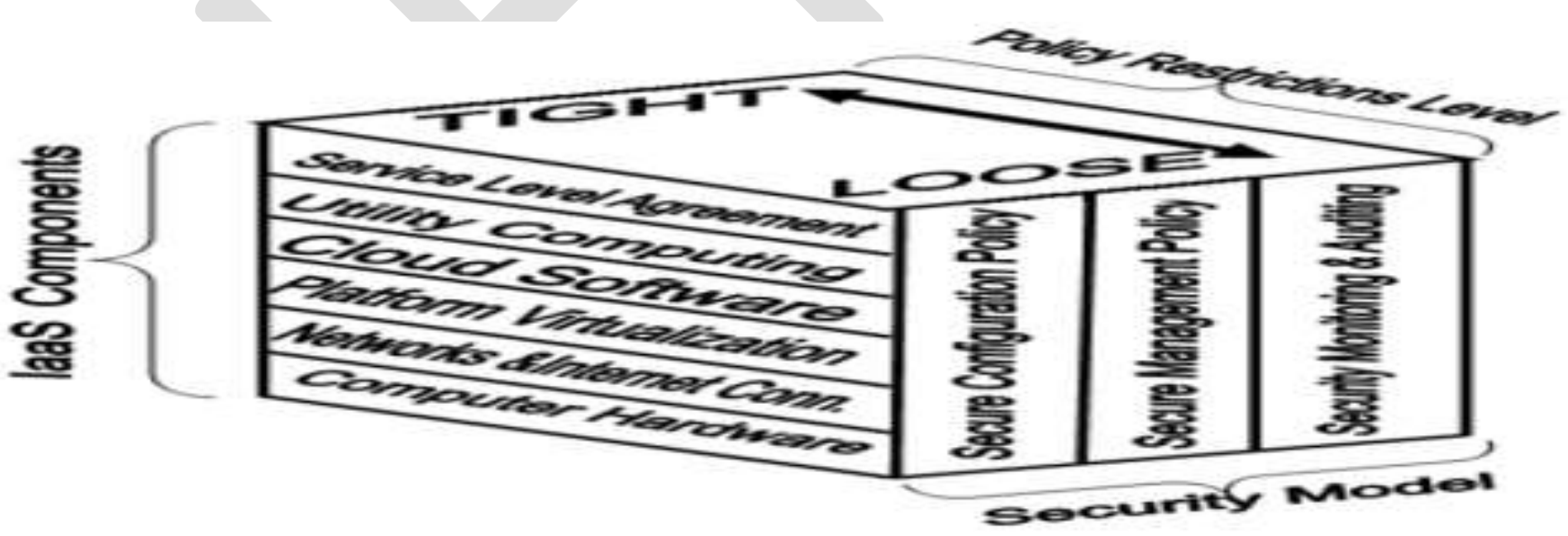

Figure 4: Security Model in IAAS

As a result of this research, we also have a security model annual (SMI) as a guide as shown in Fig $4 \%$ annual increase protection in every layer of the distribution model and discussed to assess SMI consists of three sides of the model: the model and the restriction level annual components. Cube model on the front side of the components in the annual discussions on the previous sections well. Three vertical bodies of security model where each unit covers the entire annual component. The first unit secures configuration policy (annual SLA hardware, software, or configuration in each layer guarantees a safe configuration for SCP); Typically, Miss- 
configuration events can jeopardize the entire security system. The second a protected resource management policy (management roles andprivileges control SRMP). Monitor security policy and audit the last entity (systems to track important life cycle what is restriction policy side SPMA) security model specifies the level of sanctions for entities. Customers and service providers from the loose restrictions, depending on the requirements begins to pester. Nevertheless, we hope the annual layers SMI model standardization a good start for the relationship between annual components and security requirements model indicates, And safety improvements to achieve a total secure annual system for easy individual layers.

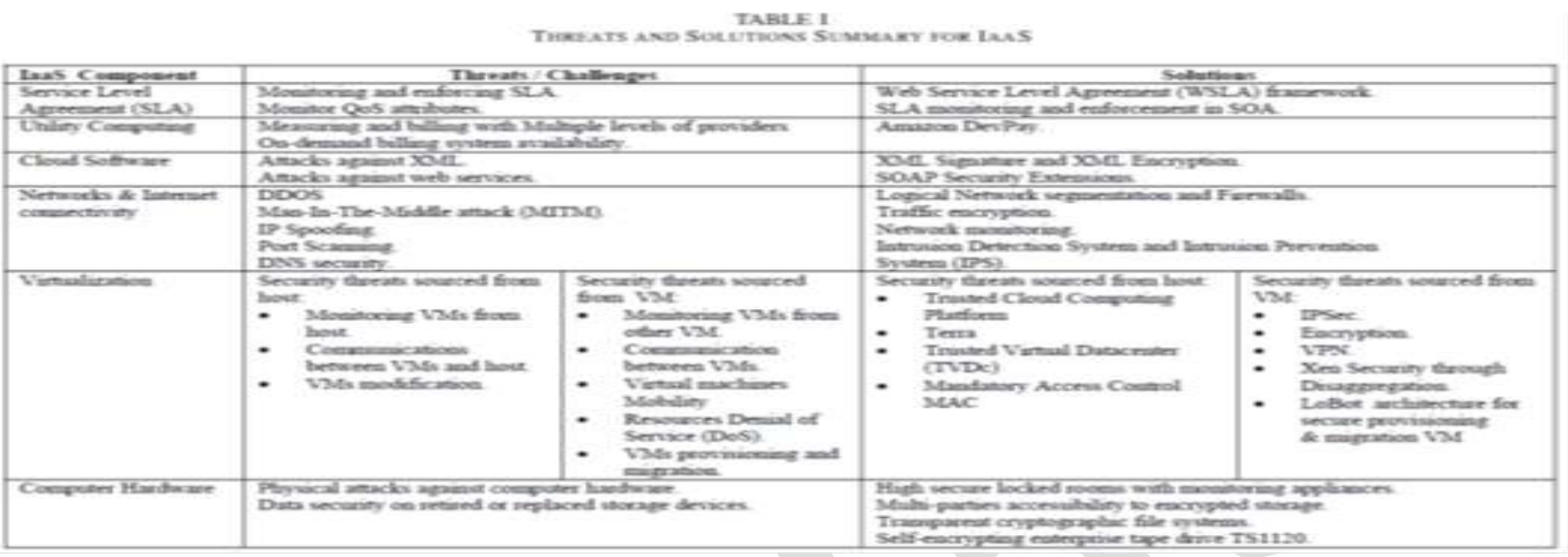

(SRMP) control management roles and privileges, monitor and audit the last entity security policy (System life cycle is important to track the SPMA). Restrictions for the security restriction policy side specify the level of model entities. Restriction level loose provider, client and service requirements based on tight then begin to also the layers SMI model standardization a good start for the relationship between annual components and security requirements model indicates, and safety improvements to achieve a total secure annual system for easy individual layers.

\section{ACKNOWLEDGMENT}

I want to say thank you to my family. I am very grateful to my College of Medicine,Baghdad University, Iraqto support me during my Msc. in computer science in BharatiVidyapeethdeemd,YashwantraoMohite College(Arts, Commerce, Science)Pune,and other faculty and associates of computer science department who are directly or indirectly helped me for this work. This work is to get on a scientific level and raise the level of education at the University of Baghdad in Iraq.

\section{CONCLUSION}

In this paper we have different layers as a service infrastructure; we also discuss about security to provide a public key infrastructure (PKI) is that each layer we can discuss in this paper. SLA only services provided and if this discount services agreement were found, but in fact to meet our deficit did not help customers about the discount. In this paper we discuss security holes associated with the annual implementation. Security issues here in addition to the recently proposed solutions for each annual component of safety concern

\section{REFERENCES:}

[1].M. Jensen, J. Schwenk, N. Gruschka, and L. Lo Iacono, On Technical Security Issues in Cloud Computing . IEEE, 2009.

[2]. Greg Boss, Padma Malladi, Denis Quan, Linda Legregni, Harold Hal 1, "Cloud Computing", http://www.ibm.com/developerswork/websphere/zones/hip ods/library.html, October 2007, pp. 4 - 4

[3]G. Frankova, Service Level Agreements: Web Services and Security, ser. Lecture Notes in Computer Science. Berlin, Heidelberg: Springer Berlin Heidelberg, 2007, vol. 4607.

[4]. "Service Level Agreement and Master Service Agreement", http://www.softlayer.com/sla.html, accessed on April 05, 2009.

[5]. S. Berger, R. Caceres, D. Pendarakis, R. Sailer, E. Valdez, R. Perez, W. Schildhauer, and D. Srinivasan, "Security for the cloud infrastrcture: trusted virtual data center (TVDc)." [Online]. Available: www.kiskeya.net/ramon/work/pubs/ibmjrd09.pdf

[6]. http://www.cloudsecurity.org, accessed on April 10, 2009. 
International Journal of Engineering Research and General Science Volume 2, Issue 6, October-November, 2014

ISSN 2091-2730

[7]. "Sampling issues we are addressing", http://cloudsecurityalliance.org/issues.html\#15, accessed on April 09, 2009.

[8]. MikeKavis,"Real time transactions in the cloud",

http://www.kavistechnology.com/ blog/?p=789, accessed on April 12, 2009.

[9]. "Secure group addresses cloud computing risks",

http://www.secpoint.com/security - group - addresses -

cloudcomputing - risks.html, April 25, 2009.

[10]. "Service Level Agreement Definition and contents", http://www.service - level - agreement.net, accessed on March 10, 2009.

[11] "Cloud security alliance: Security guidance for critical areas of focus in cloud computing v2.1," Dec 2009. Available at: www.cloudsecurityalliance.org.

[12]. "WesamDawoud, Ibrahim Takouna, ChristophMeinel Infrastructure as a Service Security 